\title{
Valoración de la utilidad de la materia de probabilidad y estadística en la carrera de IGE en el ITSOEH
}

\section{Valuation of the usefulness of probability and statistics in IGE's career at ITSOEH}

GARCÍA-ROJAS, Jesús Alberto†, RODRÍGUEZ-AGUILAR, Raquel, OROPEZA-MENDEZ, José Martín y ALVAREZ-CRUZ, Arminda

Instituto Tecnológico Superior del Occidente del Estado de HidalgoIngeniería en Gestión Empresarial. Ingeniería en Tecnologías de la Información y Comunicación. 42700 Mixquiahuala, Hgo.

ID 1er Autor: Jesús Alberto, García-Rojas / ORC ID: 0000-0002-0292-0789, Researcher ID Thomson: I-9924-2018, CVU CONACYT ID: 397590

ID $1^{\mathrm{er}}$ Coautor: Raquel, Rodríguez-Aguilar

ID $2^{\text {do }}$ Coautor: José Martín, Oropeza-Mendez / ORC ID: 0000-0003-3602-6942, Researcher ID Thomson: P-57292018, CVU CONACYT ID: 883665

ID $3^{\text {er }}$ Coautor: Arminda, Alvarez-Cruz

DOI: $10.35429 / J O T E .2019 .7 .3 .1 .7$

Recibido 10 de Enero, 2019; Aceptado 20 de Marzo, 2019

\section{Resumen}

La SICES en el año 2017 solicita al ITSUR y a deSoft el desarrollo de la plataforma SUREDSU, plataforma web que se desarrollará para detectar las expectativas educativas de los estudiantes de las escuelas medio superior del estado de Guanajuato. Con este proyecto, deSoft, enfrenta nuevos retos: realizar software para un cliente externo, desarrollar una plataforma dirigida a usuarios de entre 17 y 22 años, y además desarrollar un software con un alto nivel de concurrencia. Los integrantes de deSoft, comparten un gran interés por la calidad en la generación de software, su estrategia de calidad es la certificación en el modelo CMMI-DEV la cual se logró en el año 2013 y la cual involucra la aplicación de pruebas de software. Para superar los retos a los que se enfrenta con la plataforma SUREDSU, se han aplicado pruebas de sistema: estrés, usabilidad, funcionalidad, interfaz y de validación de datos. Logrando identificar los defectos antes de la entrega del software y con esto cubrir las expectativas del cliente y de los usuarios finales.

\begin{abstract}
SICES in 2017 requests ITSUR and deSoft to develop the SUREDSU platform, a web platform that will be developed to detect the educational expectations of students in the upper secondary schools of the state of Guanajuato. With this project, deSoft faces new challenges: making software for an external client, developing a platform aimed at users between 17 and 22 years old, and also developing software with a high level of concurrency. The members of deSoft, share a great interest in the quality of software generation, their quality strategy is certification in the CMMI-DEV model which was achieved in 2013 and which involves the application of software tests. To overcome the challenges faced with the SUREDSU platform, system tests have been applied: stress, usability, functionality, interface and data validation. Achieving identify the defects before delivery of the software and thereby meet the expectations of the client and end users.
\end{abstract}

Quality, Test, Bug

Calidad, Prueba, Defecto

Citación: GARCÍA-ROJAS, Jesús Alberto, RODRÍGUEZ-AGUILAR, Raquel, OROPEZA-MENDEZ, José Martín y ALVAREZ-CRUZ, Arminda. Valoración de la utilidad de la materia de probabilidad y estadística en la carrera de IGE en el ITSOEH. Revista de Educación Técnica. 2019. 3-7: 1-7.

$\dagger$ Investigador contribuyendo como primer autor. 


\section{Introducción}

La competencia que se vive en la actualidad con respecto a la rapidez con la que se toman decisiones en el mercado, que es cada vez tiene una mayor competitividad, hace que la estadística cobre vida; por otro lado se puede observar la apatía que muestran los estudiantes al platicarles la importancia del trabajo de la materia de probabilidad y estadística descriptiva, lo cual lleva a concluir que no le encuentren una utilidad a este conocimiento. Es por ello que el profesionista debe inmiscuirse y atreverse a redactar y escribir artículos científicos, pues no solo se trata de mostrar información por mostrar, sino que se trata también de dar argumentos válidos que respalden el valor de los datos, esta cuestión es vital para la vida de las empresas, ya que si se toman decisiones incorrectas pueden llevar a la empresa a la quiebra y como consecuencia a una crisis económica para el municipio en que se presente.

Una actividad clave de la materia de probabilidad y estadística es ayudar a la organización de información para posteriormente hacer el análisis correspondiente mediante una demostración de graficas que puedan ser fácilmente interpretadas por sus lectores. El respaldo que proporciona la estadística para sustentar la toma de decisiones es considerablemente importante, ya que facilita detectar tendencias, frecuencias, eventos, etc.; cuando hablamos de la probabilidad nos referimos a que es un fenómeno que puede o no suceder y que ayuda a observar las posibilidades de éxito o fracaso de un evento a medir, esta cuestión puede dar mayor certidumbre en la predicción de eventos para que las empresas puedan prevenirlos.

El objetivo del presente artículo es que los estudiantes puedan identificar el riesgo de no contar con los conocimientos que corresponden a esta materia tan importante para su carrera profesional, otro factor de gran afectación es la falta de conocimientos de redacción y de ortografía, pues no es nuevo este tema, pero deja ver que si se muestran trabajos con baja calidad en la redacción podrían ser poco profesionales; por lo que se requiere una fuerte campaña de difusión como es el caso del presente artículo de investigación.

\section{La probabilidad y estadística}

La estadística brinda una herramienta para el análisis e interpretación de un conjunto de datos con la finalidad de:

- Estadística: Aporta, herramientas desde el análisis e interpretación de datos (estadística descriptiva), al proceso de predicción y toma de decisiones (estadística inferencial). Analiza o procesa conjuntos de datos numéricos, estudia las funciones decisorias estadísticas, fenómenos conjuntos para revelar los fundamentos de su desarrollo y para tal estudio se sirve de índices generalizadores (valores, medios, relaciones, porcentajes, etc.).

- La estadística descriptiva: Recolecta, analiza y caracteriza un conjunto de datos (peso de la población, beneficios diarios de una empresa, temperatura mensual,...) con el objetivo de describir las características y comportamientos de este conjunto mediante medidas de resumen, tablas o gráficos.

- Estadística inferencial: Busca deducir propiedades de una población estudiada, explica ciertas características a partir de los datos obtenidos. La estadística inferencial implica obtener las conclusiones correctas de un análisis estadístico realizado mediante estadística descriptiva.

- Probabilidad: Es la posibilidad que existe entre varias posibilidades, que un hecho o condición se produzcan, mide la frecuencia con la cual se obtiene un resultado en oportunidad de la realización de un experimento sobre el cual se conocen todos los resultados posibles gracias a las condiciones de estabilidad que el contexto supone de antemano.

- Promedio: Se suman todos los valores de la variable $\mathrm{x}$ y se dividen entre $\mathrm{n}$ el número de esos valores.

- Mediana: Es el valor ocupado por la posición central cuando los datos se ordenan de acuerdo con su magnitud.

- Moda: Es el valor de x que ocurre con mayor frecuencia. 
- Muestreo: Es la técnica para la selección de una muestra a partir de una población estadística.

\section{Importancia de la Estadística.}

- Es un factor claro en las predicciones y tomas de decisiones a partir de datos observados.

- Es imprescindible que el investigador tenga conocimientos básicos sobre estadística para no llegar a conclusiones equivocadas.

- Proporciona alternativas cuantitativas al juicio personal, de forma que realice conclusiones objetivas.

Histogramas: es una herramienta de tipo estadístico, concretamente de la estadística descriptiva. La estadística auxilia a la investigación al tratar con los siguientes temas.

1. La colecta y compilación de datos.

2. El diseño de experimentos.

3. La medición de la valoración, tanto de datos experimentales como de reconocimientos y detección de causas.

4. El control de la calidad de la producción.

5. La determinación de parámetros de población y suministro de varias medidas de exactitud y precisión de esas estimaciones.

6. La estimación de cualidades humanas.

7. La investigación de mercados, incluyendo escrutinios de opinión emitidas.

8. El ensayo de hipótesis respecto a poblaciones.

9. El estudio de la relación entre dos o más variables.

\section{La encuesta}

A continuación, se muestra la encuesta que se aplicó a los estudiantes de la carrera de Ingeniería en Gestión Empresarial, con un total de 316 , en la cual se determinó mediante la siguiente formula:

\section{Población, muestra y método de recolección de información}

La técnica de recolección de datos a utilizar como elemento esencial para esta investigación ha sido la encuesta, que se ha diseñado en específico para la obtención de la información que permitirán identificar el entendimiento e impacto de la materia de probabilidad y estadística descriptiva donde se calculó una muestra para la aplicación de la misma (Fórmula 1), que se anexa a continuación: Para la obtención de la muestra se ha utilizado la siguiente Fórmula (1):

$$
n=\frac{K^{2} * N * p * q}{e^{2}(N-1)+K^{2} * p * q}
$$

Figura 1 Fórmula para determinar muestras en poblaciones finitas

Fuente: (Grant \& Leavenworth, 2005)

La fórmula utilizada para el establecimiento de la muestra es la siguiente teniendo como universo 316 alumnos inscritos en la carrera de Ingeniería en Gestión Empresarial de ITSOEH (Instituto Tecnológico Superior del Occidente del Estado de Hidalgo) en la carrera de IGE:

Dónde:

$$
\begin{aligned}
& \mathrm{N}=\text { Población }(316) \\
& \mathrm{K}^{2}=\text { Coeficiente de confianza }(90 \%) . \\
& \mathrm{e}^{2}=\text { Error admisible }(10 \%) \\
& \mathrm{p}=\text { Probabilidad a favor }(50 \%) . \\
& \mathrm{q}=\text { Probabilidad en contra }(50 \%) .
\end{aligned}
$$

El resultado de aplicar la formula fue de 56 encuestas. La encuesta trato acerca del conocimiento básico de probabilidad y estadística, así como la forma de enseñanza que más les beneficia.

La Figuras 2 y 3 muestran la primer parte de la encuesta aplicada. 


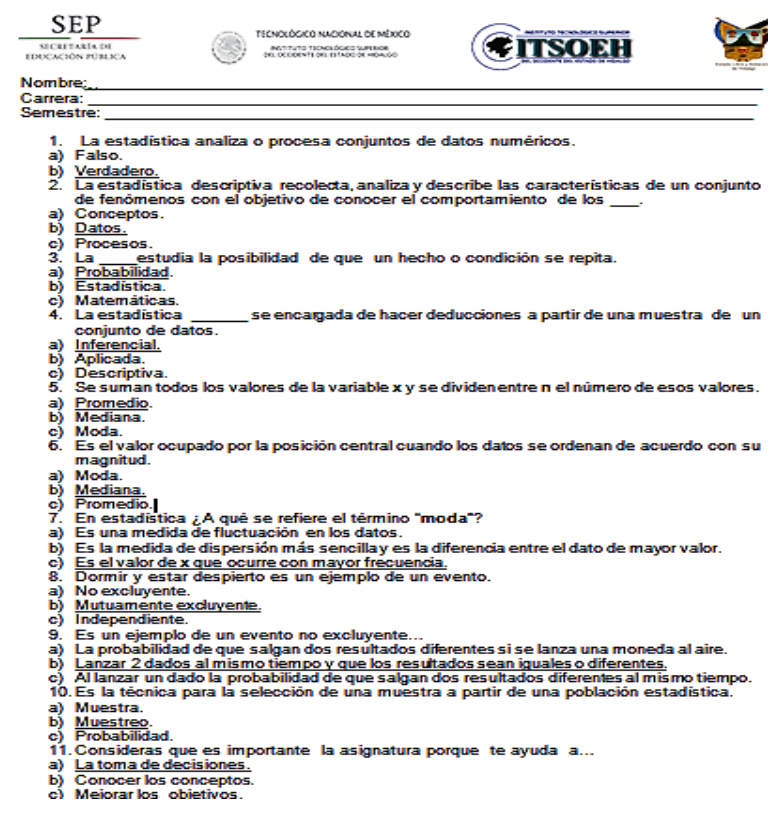

Figura 2 Primera parte de la encuesta

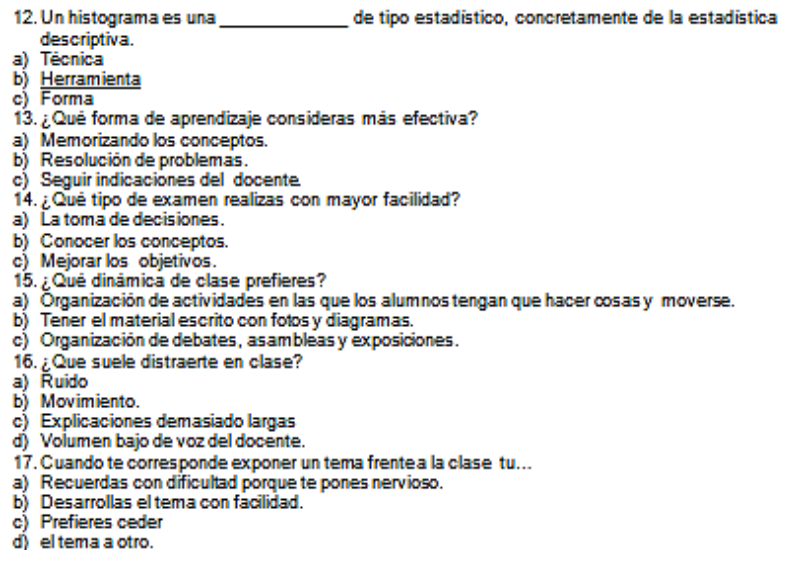

Figura 3 Segunda parte de la encuesta

\section{El sentimiento del estudiante}

A manera de sondeo se platicó con los alumnos de la carrera de IGE, en la que algunos alumnos en su mayoría comentan que es muy importante que se les tome en cuenta, pues son los alumnos el principal elemento del proceso educativo, por otro lado comentaron que es importante difundir el impacto de este artículo, pues permitirá a un mayor número de alumnos conocer la importancia de la materia de probabilidad y estadística descriptiva y que como prueba sirva este artículo, donde precisamente cobra vida este análisis numérico de la problemática planteada, que a su vez permitirá fortalecer el valor de la toma de decisiones, que en este caso el docente autor de este articulo tomara las decisiones pertinentes con su clase de la materia de probabilidad y estadística descriptiva, dando con ello el interés al alumno y sobre todo dar a conocer la importancia del contenido temático de la materia.

\section{Metodología a desarrollar}

En esa fase de desarrollo se verá reflejado el impacto de la obtención de datos, del tiempo y el estado emocional del alumno, impactando de manera directa en los resultados. (Hernández Sampieri , Fernández Collado, \& Baptista Lucio, Metodología de la investigación, 2014)

Además de utilizar el método cuantitativo y cualitativo con la aplicación de encuestas con el fin de conocer las opiniones de distintos alumnos (Figura 4).

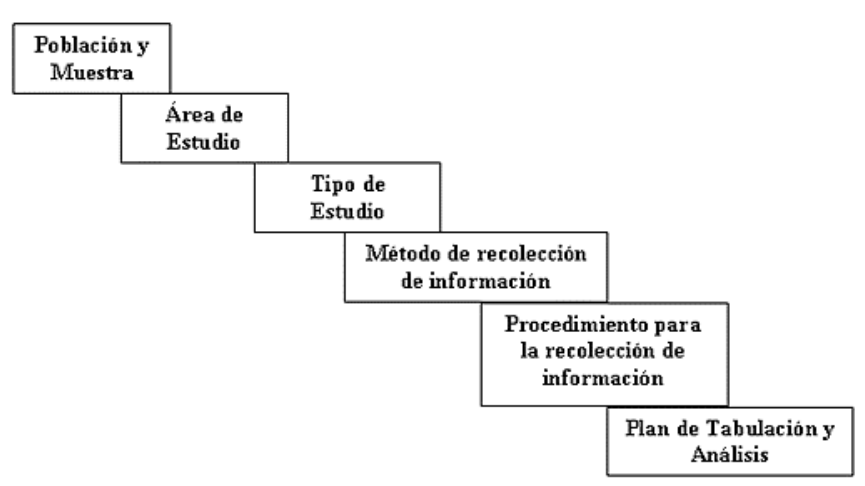

Figura 4 Procedimiento para el análisis cuantitativo de información

\section{Resultados}

Para la pregunta 1, sobre el concepto de estadística, el $100 \%$ de los alumnos encuestados contestaron de manera adecuada, dejando ver que tienen un conocimiento básico sobre la teoría de la estadística. En la pregunta 2 , el $96 \%$ de los alumnos contestaron de manera adecuada poniendo claro que se manejan datos dentro de la estadística descriptiva.

Para la pregunta 3, el $91 \%$ de los alumnos contestaron de manera adecuada, pues contestaron que utilidad tiene la probabilidad, la cual es de predecir hechos o condiciones. En la pregunta 4, el 59\% de los alumnos encuestados contestaron de manera adecuada o correcta, ya que existe la confusión acerca de lo que significa la estadística descriptiva y la inferencial, tienen una gran diferencia.

Para la pregunta 5, el 91\% contesto de manera correcta, dentro de la pregunta que se realizó qué acerca de la definición de un promedio, esto deja ver que tiene $\mathrm{n}$ las bases teóricas. Para la pregunta 6 , el $95 \%$ de los encuestados contesto de manera adecuada, en la cual se menciona el concepto de lo que es una mediana. 
Para la pregunta 7, el 95\% contesto de manera adecuada contestando el concepto de lo que es una moda. En la pregunta 8, la cual fue una pregunta básica sobre eventos mutuamente excluyentes, pregunta que hasta suena lógica, ya que existen 2 elementos independientes de si, por lo que el $34 \%$ de los estudiantes respondieron correctamente, lo cual ratifica que la lógica se tiene que reforzar en los estudiantes. En la pregunta 9, pregunta que trataba sobre un ejemplo de un evento no excluyente, un $46 \%$ de los estudiantes contestaron adecuadamente, dado que en esta pregunta la palabra clave es igual, ya que es un elemento incluyente.

En la pregunta 10, pregunta acerca de lo que es un muestreo resulto que un $70 \%$ contesto de manera adecuada ya que es un tema con mayor arraigo en la carrera de gestión empresarial. En la pregunta 11, acerca del factor clave de la probabilidad y estadística que sirven para la toma de decisiones, contestaron de manera correcta un $96 \%$ lo cual ratifica la importancia final que tiene esta materia.

En la pregunta 12, el $73 \%$ que es mayoría contesto de manera correcta que es un histograma. En la pregunta 13, el $88 \%$ que es mayoría contesto la manera correcta de aprender es resolviendo problemas. En la pregunta 14 , el $48 \%$ donde hubo casi un empate con la misma cifra entre la toma de decisiones y conocer los conceptos, esto da a conocer lo que mejor representa al alumno cuando presenta un examen. En la pregunta 15, el $60 \%$ que es mayoría respondió que la dinámica que las les gusta de las clases son las clases dinámicas donde implica que el alumno se mueva. Donde también se acerca con el $32 \%$ donde los alumnos prefieren que sea la clase por medio de fotos y diagramas.

En la pregunta 16 , el $42 \%$ que es mayoría responde que el ruido es lo que más lo distrae en clases, mientras el $32 \%$ comenta que el movimiento los distrae de clases.

En la pregunta 17, el $80 \%$ que es mayoría comenta que cuando le toca exponer un tema frente a clases el mismo alumno desarrolla el tema con mayor facilidad. Por eso es importante que los alumnos expongan con mayor frecuencia un tema.

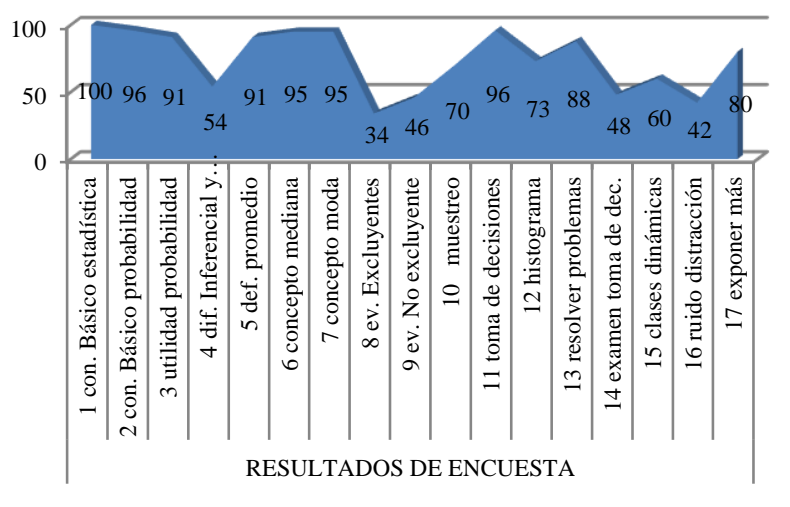

Gráfico 1 Resultados de encuesta de valoración de la materia de probabilidad y estadística descriptiva en el ITSOEH

ojiva-frecuencia acumulada

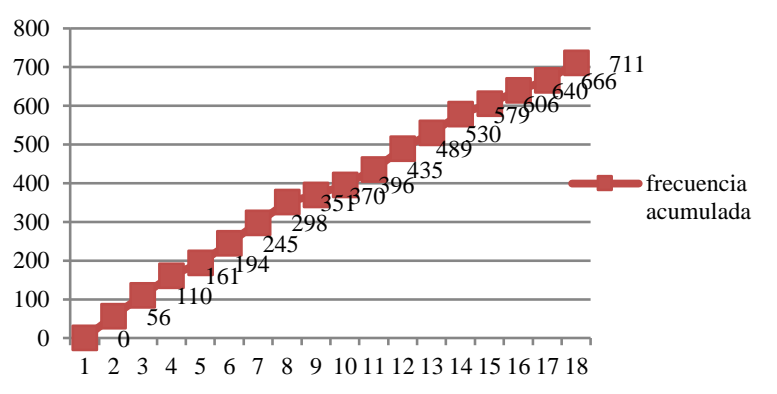

\section{Gráfico 2 Ojiva}

El Gráfico2 muestra el ejemplo de ojiva de frecuencia acumulada, que muestra la tendencia de crecimiento de los porcentajes presentados en los resultados de encuesta, dejando ver donde existe una mayor diferencia entre cada punto.

\begin{tabular}{|lr|}
\hline \multicolumn{1}{|c|}{ Tendencia central } & \multicolumn{2}{c|}{ Resultado } \\
\hline Mediana & 80 \\
\hline Moda & 96 \\
\hline Desviación estándar & 22.40 \\
\hline Media & 66.2 \\
\hline
\end{tabular}

Tabla 1 Medidas de tendencia central

En la Tabla 1 se muestran indicadores de tendencia central, que de acuerdo a los resultados obtenidos en la encuesta se puede calcular que la mediana en porcentaje es del $80 \%$, lo cual deja ese porcentaje de aprovechamiento como indicador general sobre nuestro estudio. Por otro lado el dato más repetido o moda fue de $96 \%$. La desviación estándar fue de 22.4 y la media fue de 66.2 , esto nos indica que tanto se separan los datos obtenidos con respecto a la media. 


\section{Agradecimiento}

Agradecemos a los alumnos de Ingeniería en Gestión Empresarial por su contribución a la elaboración del presente artículo, así como al ITSOEH por las facilidades y apoyo económico para la publicación del mismo.

\section{Conclusiones}

Concluyendo el presente artículo de investigación se comenta que los alumnos tienen buenos conocimientos teóricos sobre la probabilidad y estadística, lo cual es bueno para poder abordar temas de mayor complejidad, donde puedan tomar decisiones como futuros profesionistas, los estudiantes muestran que conocen la utilidad de la probabilidad, conocen como se calcula un promedio, mediana y moda, los cuales son términos comunes en la probabilidad y estadística, por lo que el alumno conoce en términos positivos lo que es un muestreo. (Grafico 1). Llegando al punto que llama la atención donde los indicadores son bajos, que son que los alumnos no diferencian entre la estadística inferencial y descriptiva, este indicador es bajo, además desconocen los eventos incluyentes y excluyentes, lo cual también tiene un indicador bajo, dejando ver que son temas que abordar con mayor atención por parte de los docentes.

Los alumnos conocen de manera general que es un histograma. Pasando a los puntos de la docencia de la clase de probabilidad y estadística descriptiva, se concluye que los alumnos en su mayoría les gustan que se incluyan ejercicios para resolver problemas, así como también les fortalece que expongan temas, pero por otro lado les molesta el ruido que se genera dentro y fuera de las aulas.

Los alumnos reconocen de manera regular que los exámenes fortalecen el ejercicio de toma de decisiones. A los alumnos les gustan las clases dinámicas y con el manejo de diagramas de flujo e imágenes.

Como se puede observar, es necesario reconocer los puntos fuertes que muestra este artículo, como son conocimientos básicos de probabilidad y estadística, medidas de tendencia central, muestreo.
Por otro lado una área de oportunidad es profundizar en temas como la estadística inferencial y descriptiva para que reconozcan su diferencia, pero para poder ayudarles a los alumnos en esta tarea del aprendizaje, fomentemos la reducción del ruido en clases y aumentemos el material visual y dinámico de las clases, fomentando la exposición de los temas, para que los alumnos refuercen los mismos. Como se puede concluir mediante este artículo se puede observar que debemos conocer términos teóricos en primera instancia, para después por medio de ejemplos entendamos las diversas herramientas de análisis de datos de la probabilidad y estadística descriptiva, haciendo énfasis en la estadística inferencial y descriptiva para fortalecer conocimientos, llevando problemas reales al alumno $y$ fomentando buenas practicas pedagógicas podremos aumentar el aprendizaje significativo del alumno, así como se podrá fortalecer el desarrollo de competencias y habilidades que harán del alumno un profesionista con mayor competencia con respecto al estudiante promedio, porque esta materia la utilizaran toda su vida, ya sea personalmente, académicamente.

\section{Referencias}

Levin, R. I., Rubin, D. S., \& Samaniego, A. H. F. (1996). Estadística para administradores (No. 519.5 L47Y 1994). Prentice-Hall Hispanoamericana.

Ross, S. M. (2007). Introducción a la estadística. Reverté.

Dixon, W. J., Massey, F. J., \& García, S. R. (1966). Introducción al análisis estadístico (No. QA276. D59 1965.). SA Madrid: Ediciones del Castillo.

Caballero, W. (1975). Introducción a la estadística (Vol. 28). IICA.

Box, G. E., Hunter, J. S., \& Hunter, W. G. (2005). Estadística para investigadores: Introducción al diseño de experimentos, análisis de datos y construcción de modelos. Reverté.

Chao, L. L., \& Castaño, J. M. (1993). Estadística para las ciencias administrativas (Vol. 3). México: McGraw-Hill. 
Batanero, C., \& Godino, J. (2005). Perspectivas de la educación estadística como área de investigación. Líneas de investigación en Didáctica de las Matemáticas, 203-226.

Ramírez, T. (1999). Cómo hacer un proyecto de investigación. $126 \quad 1 \quad$ CIC-UCAB/0022 20031106 GPM.

Batanero, C., \& Díaz, C. (2011). Estadística con proyectos. Granada: Universidad de Granada.

Sabadías, A. V. (1995). Estadística descriptiva e inferencial (Vol. 8). Univ de Castilla La Mancha.

Parra, J. M. (1995). Estadística descriptiva e inferencial I. Recuperado de: http://www. academia.

edu/download/35987432/ESTADISTICA_DES

CRIPTIVA_E_INFERENCIAL.pdf.

Canavos, G., Meyer, P., Spiegel, M., \& Mendenhall, S. (1988). Probabilidad y estadística. LICENCIATURA EN INGENIERÍA EN INFORMÁTICA, 28.

Montgomery, D. C., Runger, G. C., \& Medal, E. G. U. (1996). Probabilidad y estadística aplicadas a la ingeniería (No. 968-18-5914-6. 01-A1 LU. AL-PyE. 1.). Mexico DF, México: McGraw-Hill.

Miller, I., \& Freund, J. E. (1973). Probabilidad y estadística para ingenieros. Reverté.

Triola, M. F. (2004). Probabilidad y estadística. Pearson educación.

Meyer, P. L., Campos, C. P., \& Cuéllar, G. A. (1973). Probabilidad y aplicaciones estadísticas (No. QA273. 25. M49 1973.). Bogotá: Fondo Educativo Interamericano. 